ISSN: 2174-5609

DOI. http://dx.doi.org/10.14198/INTURI2016.12.08

TrT Investigaciones

MURín TICAS

\title{
Contratación on line de servicios turísticos y paquetes dinámicos de turismo ${ }^{1}$
}

\author{
Altea Asensi Meras \\ Universidad de Alicante, España \\ altea.asensi@ua.es
}

\section{RESUMEN}

La implementación de las nuevas tecnologías o TIC's en el sector turístico se ha visto favorecida, en los últimos años, por las posibilidades que Internet ofrece de poder comprobar instantáneamente la existencia real del proveedor del servicio, la veracidad del servicio prestado o las condiciones en que se presta. El aumento de la contratación electrónica de servicios turísticos exige que los proveedores de estos servicios y los turistas tengan en cuenta las diferentes normas jurídicas que resultan de aplicación en el ámbito de los servicios de la sociedad de la información, principalmente, la Ley 34/2002, de 11 de julio, de Servicios de la Sociedad de la Información y de Comercio Electrónico (LSSI), las normas reguladoras del consumo. Por otra parte, la nueva Directiva 2015/2302 del Parlamento Europeo y del Consejo, de 25 de noviembre de 2015, relativa a los viajes combinados y a los servicios de viaje vinculados ofrece una nueva regulación aplicable a los viajes combinados y a los denominados "servicios de viaje combinado" que resultan de aplicación a las nuevas formas de contratación on line de servicios turísticos.

Palabras Clave: Comercio electrónico, contratación electrónica, servicios turísticos, proveedor del servicio sociedad de la información, paquetes dinámicos de turismo.

\footnotetext{
${ }^{1}$ El presente trabajo se realiza en el marco del Proyecto de Investigación AICO/2015/047 de la Generalitat Valenciana.
} 


\title{
On-line contracting of tourism services and dynamic packages of tourism
}

\begin{abstract}
In recent years, the implementation of new technologies or ICTs in the tourism sector has benefitted from the power of the Internet to enable the instant checking of whether a service provider exists or the veracity and conditions of the service. The increase of ecommerce in tourist services requires the providers of these services and the tourists to take into account the different legal standards which apply to the information society services, particularly Law 34/2002 of 11 July, on Services of the Information Society and Electronic Commerce (LSSI), the rules of consumption. Furthermore, the new Directive 2015/2302 of the European Parliament and of the Council of 25 November 2015 on package travel and linked travel arrangements provides new regulations for package travel and the so-called "package services " which apply to new forms of online tourism service procurement.
\end{abstract}

Key words: E-commerce, e-procurement, tourism services, service provider information society, dynamic tourism packages.

\section{INTRODUCCIÓN}

El empleo de Internet otorga a las empresas y destinos turísticos la posibilidad de poner a disposición de un gran número de consumidores las ofertas de productos o servicios turísticos y su adquisición en el ámbito del comercio electrónico. La relación contractual que se celebra entre el turista y el proveedor del servicio turístico por Internet permite incorporar numerosas ventajas tanto para las empresas que intervienen en el sector turístico como para los turistas (Márquez Lobillo, 2011, p. 2011). Mientras que turista puede obtener una mayor cantidad de información sobre los servicios turísticos y comparar los precios y productos; el proveedor de servicios turísticos puede conocer mejor las necesidades reales de cada cliente con la finalidad de ofrecer productos personalizados y lograr una expansión económica de su negocio.

En la actualidad, las posibilidades que ofrece Internet de poder comprobar instantáneamente la existencia real del proveedor del servicio o la veracidad del servicio prestado y las condiciones en que este se presta, han contribuido a aumentar la confianza de los consumidores en la información o en la utilización de la vía electrónica para la celebración de los correspondientes contratos de prestación de servicios turísticos. Por otra parte, la creación de plataformas seguras de pago ha permitido que el turista contemporáneo utilice la Red tanto para buscar información como para adquirir en línea los productos y servicios ofertados en el comercio electrónico (Batuecas Caletrío, 2013, p. 52).

Un tratamiento correcto de la contratación electrónica de servicios turísticos exige poner en relación las diferentes normas jurídicas. Con la finalidad de elevar la garantía y la seguridad de las transacciones efectuadas por medios electrónicos, el legislador español aprobó la Ley 34/2002, de 11 de junio, de Servicios de la Sociedad de la Información y del Comercio electrónico (LSSI) por medio de la cual se incorpora en el sistema jurídico español la Directiva 2000/31/CE, de 8 de junio de 2000 sobre comercio electrónico (Cavanillas Múgica, 2001, p. 4). Esta norma resulta directamente aplicable en el ámbito de la 
contratación electrónica de servicios turísticos, en tanto esta materia no se encuentra expresamente excluida de su ámbito de aplicación, ni sometida a la regulación de una normativa específica.

Sin embargo, la normativa sobre comercio electrónico no es la única aplicable en este ámbito ya que, tal y como se establece en la LSSI, "Ios contratos electrónicos se regirán por lo dispuesto en este Título, por los Códigos Civil y de Comercio y por las restantes normas civiles o mercantiles sobre contratos, en especial, las normas de protección de los consumidores y usuarios y de ordenación de la actividad comercial". De esta forma, no sólo será de aplicación en el ámbito de la contratación electrónica la normativa sobre comercio electrónico, sino también las generales sobre contratos, y en especial, las normas de protección de los consumidores, contenidas en el Real Decreto Legislativo 1/2007, de 16 de noviembre, por el que se aprueba el texto refundido de la Ley General para la Defensa de los Consumidores y Usuarios y otras leyes complementarias (Cruz Rivero, 2009, p. 25).

Por otro lado, cabe señalar que la Constitución Española de 1978 establece que las Comunidades Autónomas podrán asumir competencias en materias relativas a la "promoción y ordenación del turismo en su ámbito territorial". En este sentido, deberán tenerse en cuenta la normativa autonómica que, en la actualidad, realicen referencias expresas a los proveedores que intervienen en la comercialización de productos y/o servicios turísticos por medios electrónicos. Asimismo, y siempre que proceda, deberán tenerse en cuenta la posible legislación turística específica aplicable al supuesto concreto, como es, en particular, la Ley 4/2012, de 6 de julio, de contratos de aprovechamiento por turno de bienes de uso turístico, de adquisición de productos vacacionales de larga duración, de reventa y de intercambio y normas tributarias (Batuecas Caletrío, 2013, p. 51).

Por último, al ser una modalidad contractual que incluye condiciones generales de la contratación, la contratación electrónica de servicios turísticos se encuentra dentro de la categoría de los contratos de adhesión, que se caracterizan por ser contratos no negociados individualmente en los que el cliente se limita a aceptar el contenido impuesto por el prestador del servicio que organiza esta modalidad de mercados (González de la Alaiza Cardona, 2010, p. 1705). De esta forma, a esta modalidad contractual también le resultará de aplicación el régimen aplicable a las condiciones generales de la contratación que se establece en la Ley 7/1998, de 13 de abril Ley de Condiciones Generales de la Contratación y su normativa de desarrollo (Paniza Fullana, 2012, p.15). Asimismo cabrá de tener en cuenta el régimen jurídico de las condiciones generales y las cláusulas abusivas aplicable en el ámbito de los contratos con consumidores.

\section{LOS OFERENTES EN LA COMERCIACIÓN ON LINE DE SERVICIOS TURISTICOS}

Las agencias de viajes que operan a través de Internet para ofrecer, y en su caso, comercializar productos y/o servicios turísticos, realizan una actividad de prestación de servicios de la sociedad de la información ya que se trata de una actividad consistente en la prestación de un servicio que se suministra en el marco de una actividad organizada, normalmente a título oneroso, a distancia, por medios electrónicos y a petición individual de un destinatario. En este sentido, dichas empresas turísticas tendrán la consideración de prestadores de servicios de la sociedad de la información, y estarán sometidas al estatuto 
jurídico previsto en la Ley 34/2002, de 11 de julio, de Servicios de la Sociedad de la Información y de comercio electrónico (Miguel Asensio, 2011, p. 119).

La sumisión de las agencias de viajes on line al estatuto jurídico propio de los prestadores de servicios de la sociedad de la información no supone, sin embargo, que éstas estén exentas del cumplimiento de las obligaciones que se deriven del ejercicio de esta actividad en el mercado tradicional. Esto implica, en primer lugar, que la actividad que desarrollen las agencias de viajes on line debe estar comprendida entre las actividades que, con carácter general, le corresponde realizar a las agencias de viajes que operan en el comercio tradicional, esto es, la organización o comercialización de los viajes combinados, así como en la realización de cualquier otra actividad que consista en la mediación y/o organización y en la contratación de cualesquiera otros servicios prestados por las empresas turísticas (Batuecas Caletrío, 2013, p. 53).

En segundo lugar, puesto que las agencias de viajes que operan por Internet desarrollan una actividad mercantil, cuando su forma jurídica sea la propia de una sociedad mercantil anónima o de una sociedad de responsabilidad limitada, estas empresas deberán someterse, debido a su forma jurídico mercantil, al régimen jurídico establecido en el Real Decreto Legislativo $1 / 2010$, de 2 de julio, por el que se aprueba el texto refundido de la Ley de Sociedades de Capital (LSC). Por último, las agencias de viajes que operan por Internet deberán de someterse, asimismo a las normas administrativa que les sean aplicables, en particular, en lo relativo al inicio y el ejercicio de la actividad que desarrollan.

Por otra parte, el régimen de autorización administrativa aplicable a las agencias de viajes ha sido modificado a partir de la entrada en vigor de la Ley 17/2009, de 23 de noviembre, de libre acceso a las actividades de servicios y su ejercicio, por medio de la cual se incorporó al sistema jurídico español la Directiva 2006/123/CE, de 12 de diciembre, relativa a los servicios en el mercado interior (Aurioles Martín, 2006, p. 44). La incorporación de esta normativa ha supuesto la supresión de la exigencia consistente en obtener una autorización administrativa previa para el desarrollo de la actividad turística (Melgosa Arcos, 2011, p. 49). De esta forma, la mayoría de disposiciones autonómicas contemplan únicamente la necesidad de que las agencias de viajes deban realizar una simple comunicación a la autoridad administrativa correspondiente en la que se ponga de manifiesto que se cumplen los requisitos exigidos para el inicio de la actividad y la información necesaria para el control de la actividad.

En la actualidad, la denominación "portal de viajes" se aplica no sólo para referirse a cualquier página web donde se ofrezcan servicios relacionados con el turismo sino que también se utiliza para las agencias de viajes que ofrecen todo tipo de servicios y a los modernos metabuscadores o comparadores de precios (Batuecas Caletrío, 2013, p. 57). Los metabuscadores o comparadores de precios, sin embargo, no prestan propiamente servicios turísticos ni intervienen en la comercialización de los mismos ya que esta función les corresponde solo a las agencias de viajes o centrales de reservas. Su responsabilidad en orden al correcto cumplimiento del servicio prestado deberá valorarse en función de la publicidad realizada así como de la eficacia de su algoritmo de búsqueda.

En cualquier caso, todo oferente o intermediario en la comercialización de servicios turísticos on line deberá de prestar la información requerida para llevar a cabo su correcta identificación frente al usuario de dicho servicio. En este sentido, las disposiciones sobre comercio electrónico imponen a los prestadores de servicios de la sociedad de la 
información que utilicen Internet como cauce publicitario para ofertar sus productos o servicios, el deber de suministrar a los destinatarios una serie de deberes de información con la finalidad de que éstos tengan la suficiente información sobre la agencia y sobre las características del producto o servicio que se pretende adquirir que si contratasen con una agencia de viajes que operase por medios tradicionales (Miguel Asensio, 2011, p. 120).

En primer lugar, la Ley 34/2002, de 11 de julio, de Servicios de la Sociedad de la Información y de comercio electrónico establece, en el artículo 10 LSSI, que todo prestador de servicios que opere por medios electrónicos, deberá poner a disposición de los destinatarios, los medios que les permitan acceder de forma fácil, directa y permanente al conjunto de información relacionados con la identidad de la empresa y que tiene que referirse a los siguientes datos: nombre o denominación social, residencia o domicilio del prestador de servicios, dirección de correo electrónico o cualquier otro dato que permita una comunicación directa y efectiva; datos registrales; el régimen de autorización administrativa previa o los datos relativos a la declaración responsable; datos fiscales; la información sobre el producto especialmente sobre el precio del mismo, y los códigos de conducta a los que pueda estar adherido dicho prestador de servicios.

Por medio de esta medida se pretende poner en conocimiento de los destinatarios la suficiente información acerca de la identidad del prestador de servicios que oferta o comercializa los productos o servicios por Internet y el lugar en el que se encuentra establecido. Asimismo, esta medida permite generar una mayor confianza entre los destinatarios de los servicios de la sociedad de la información en el caso de que utilicen Internet como cauce para llevar a cabo la contratación, ya que en el conjunto de la información suministrada se facilitan los datos que permiten efectuar una comunicación directa y efectiva entre el prestador de servicios y el destinatario de los mismos.

En segundo lugar, la normativa sobre la contratación a distancia contenida en el Real Decreto Legislativo 1/2007, de 16 de noviembre, por el que se aprueba el texto refundido de la Ley General para la Defensa de los Consumidores y Usuarios y otras leyes complementarias, tras su modificación por la Ley 3/2014, de 27 de marzo, establece que todo empresario deberá suministrar a los consumidores, además de los datos relativos a su identificación, la información relativa a las características esenciales del bien o servicio ofertado, así como el precio final completo del mismo. Esta referencia implica que la agencia de viajes que opere por Internet deberá hacer mención expresa en la oferta que realice en su página web, si se trata un servicio turístico suelto o un viaje combinado (Márquez Lobillo, 2011, p. 225).

En el caso de las ofertas de servicios turísticos sueltos, deberán indicarse todas las características que afecten a la prestación y a las circunstancias del mismo, es decir, la modalidad del servicio turístico, características del medio de transporte o alojamiento y toda la información esencial relativa al precio del servicio turístico así como cualesquiera otros gastos adicionales que puedan derivarse de la prestación del servicio. En el caso de que se trate de un viaje combinado, la agencia o detallista deberá disponer a favor del destinatario el folleto o programa informativo que tiene el carácter de verdadera oferta contractual y en el que se debe incluir la información relativa a las características esenciales del servicio y el precio global del mismo. 


\section{LA CONTRATACIÓN ELECTRÓNICA DE SERVICIOS TURÍSTICOS}

En la práctica, cada vez es más frecuente la contratación de un servicio turístico suelto consistente en la compra de un billete de avión o tren o en la reserva de una plaza de alojamiento por separado a través de las páginas web de las agencias de viajes que operan en Internet. No obstante, cabe señalar, que la contratación del servicio turístico puede efectuarse directamente con el proveedor del servicio turístico a través de su página web, no alterando la forma de celebración electrónica el régimen jurídico aplicable al contrato turístico que se trate, o bien puede a través de la página web de una agencia de viajes on line, en cuyo caso será preciso distinguir si la agencia de viajes oferta productos propios o si su actuación se limita a facilitar la contratación de servicios turísticos con el prestador de los mismos.

Mientras que en el primer supuesto, la agencia de viajes oferta un producto turístico y el turista lo adquiere, lo que supone que entre ellos se establece una relación jurídica directa; en el segundo supuesto, la agencia de viajes realiza labores de intermediación entre el turista y el proveedor final del servicio turístico, celebrándose en este caso dos relaciones jurídicas. La primera relación jurídica, que se celebra entre el turista con la agencia de viajes, tiene la naturaleza jurídica del contrato de mandato o comisión, por asumir la agencia de viajes la obligación de celebrar un determinado contrato por cuenta o encargo del turista que se lo ordena (art. 244 CCom y art. 1709 CC). La segunda relación jurídica, que se celebra entre el turista y el proveedor del servicio, aunque se somete al régimen jurídico aplicable al contrato turístico que se trate, precisa de la cooperación de la agencia de viajes que opera a través de esa página web.

\subsection{Relación jurídica entre el turista y la agencia de viaje que opera a través del portal web}

Las agencias de viajes, entendidas como empresas habilitadas para actividades de intermediación $y / o$ organización de servicios turísticos, han venido cumpliendo tradicionalmente una serie de funciones entre las que se encuentran la que se refiere a la medición entre los prestadores de los servicios turísticos y los turistas interesados en los mismos. En este sentido, la relación jurídica que se celebra entre el turista y la agencia de viaje que opera a través del portal web, al igual que la relación que se celebra entre la agencia on line con el prestador concreto del servicio turístico, se enmarca en el ámbito de la colaboración entre las partes puesto que ambas partes pretenden sacar provecho de su mutua colaboración.

La relación jurídica que se celebra entre la agencia de viaje que opera a través del portal web y el turista tiene la naturaleza jurídica de un contrato de mandato o comisión mercantil ya que, de conformidad con lo establecido en el artículo 244 del Código de Comercio, "se reputará comisión mercantil el mandato, cuando tenga por objeto un acto u operación de comercio y sea comerciante o agente mediador del comercio el comitente o el comisionista“. En este caso, la agencia de viajes actuará con representación cuando contrate en nombre del turista la prestación del correspondiente servicio turístico con el proveedor del mismo (art. 247 CCom) y la comisión tendrá carácter retributivo cuando el turista tenga que pagarle la correspondiente comisión a la agencia de viajes por la gestión del servicio (art. 277 CCom). 
Aceptado el mandato o comisión, la agencia de viajes deberá responder de los daños y perjuicios que su ejecución cause al turista (art. 252 CCom y art. 1718 CC). El ejemplo típico de responsabilidad surge cuando, por ejemplo, el turista se presenta en la recepción del hotel y se le comunica que no existe ninguna reserva a su nombre. No obstante, el mero retraso en el cumplimiento de del mandato por parte de la agencia de viajes no originará su responsabilidad, sino por los daños que efectivamente el retaso cause al turista y siempre que la agencia de viajes haya actuado con culpa transgrediendo sus deberes de conducta (Batuecas Caletrío, 2013, p. 58).

En el cumplimiento de su obligación, la agencia de viajes no podrá traspasar los límites que el turista haya establecido por medio del mandato, ya que en aplicación de lo dispuesto en los artículos 256 del Código de Comercio, la agencia será responsable de los daños y perjuicios que el ocasione al turista si procediera en contra de su disposición expresa. No obstante la agencia de viajes quedará exenta de toda responsabilidad si en el desempeño de su encargo se sujeta a las instrucciones recibidas por el turista o cuando, sin traspasar los límites del contrato, cumple el encargo de manera más ventajosa para el turista que la señalada por este.

La agencia de viajes que opera a través del portal web, como cualquier sujeto que administra negocios ajenos, debe rendir cuentas al turista al término de las gestiones, lo que supondrá el envío de los billetes de transporte o justificante de la reserva del alojamiento así como de aquellos otros documentos que justifiquen las operaciones realizadas, en este caso, por vía electrónica. En este sentido, la rendición de cuentas en el ámbito de la contratación electrónica de los servicios turísticos implica que la agencia de viajes que opera por medio de la página web deberá de adoptar los medios necesarios para disponer a disposición del turista los documentos que justifiquen la operación y pagos realizados.

Dada la instantaneidad de este tipo de contrataciones será poco común que la agencia de viajes pretenda renunciar a un encargo previamente aceptado. En cualquier caso, si ello se produce, dicha renuncia será posible sin que sea necesario fundamentarla en una justa causa (art. 1736 CC). Cuando ello ocurra, bastará que lo ponga en conocimiento del turista. No obstante, la agencia de viajes on line deberá indemnizar al turista por los perjuicios que este pudiera sufrir como consecuencia de la renuncia, a menos que la renuncia se haya fundado en la imposibilidad de continuar desempeñando el encargo (Batuecas Caletrío, 2013, p. 59). El perjuicio indemnizable en estos casos es el interés contractual negativo que se producirá ante la imposibilidad de la agencia de permitirle al turista llevar a cabo la correspondiente relación jurídica con el proveedor del servicio turístico.

\subsection{Relación jurídica entre el turista y el proveedor del servicio turístico}

En el supuesto en que la agencia de viajes además de realizar una actividad consistente en publicitar sus servicios turísticos por vía electrónica, ofrezca la posibilidad de adquirir, a través de su página web, servicios turísticos con el proveedor de los mismos, en este caso, el proveedor del servicio deberá de cumplir las exigencias y obligaciones de información que se exigen en la normativa sobre comercio electrónico, tanto con carácter previo a la celebración del contrato, como con carácter posterior a la celebración del mismo, con la finalidad de generar una mayor seguridad jurídica en este ámbito. 
De conformidad con lo dispuesto en el artículo 27 de la Ley 34/2002, de 11 de julio, de Servicios de la Sociedad de la Información y de comercio electrónico, con carácter previo a la celebración de contratos turísticos por medios electrónicos, la agencia de viajes que ofrezca dicha posibilidad deberá de informar a los usuarios de forma clara, comprensible e inequívoca, sobre los siguientes aspectos: los diferentes pasos técnicos que han de darse para celebrar el contrato, si la agencia va a registrar o no el contrato y si el mismo va a ser accesible; los medios técnicos que permitan identificar y corregir los errores en la introducción de los datos antes de efectuar un pedido y las lenguas oficiales utilizadas en la celebración del contrato.

Asimismo, el artículo 97 del Real Decreto Legislativo 1/2007, de 16 de noviembre, por el que se aprueba el texto refundido de la Ley General para la Defensa de los Consumidores y Usuarios, tras la modificación llevada a cabo por la Ley 3/2014, de 27 de marzo, refuerza la información precontractual que debe de suministrarse en el de los contratos a distancia cuando señala que el empresario le facilitará de forma clara y comprensible a los consumidores la información relativa a los datos relativos a su identificación, las características esenciales del bien o servicio ofertado, así como el precio final completo del mismo. Esta referencia implica que la agencia de viajes que opere por Internet deberá hacer mención expresa en la oferta que realice en su página web de todas las características que afecten a la prestación y a las circunstancias del mismo, incluida toda la información esencial relativa al precio y cualesquiera otros gastos adicionales que puedan derivarse de la prestación del servicio.

En este sentido, el artículo 95 del Real Decreto Legislativo 1/2007, de 16 de noviembre, por el que se aprueba el texto refundido de la Ley General para la Defensa de los Consumidores y Usuarios, tras su modificación llevada a cabo por la Ley 3/2014, de 27 de marzo, ha introducido expresamente esta previsión cuando señala que los servicios de intermediación en los contratos a distancia, incluidos los prestadores de servicios de intermediación de la sociedad de la información, están obligados a procurar, en la medida de sus posibilidades y con la diligencia debida, que éstos respeten los derechos que este título reconoce a los consumidores y usuarios y cumplan las obligaciones que en él se les imponen (Batuecas Caletrío, 2013, p. 62).

De conformidad con lo dispuesto en el artículo 28 de la Ley 34/2002, de 11 de julio, de Servicios de la Sociedad de la Información y de comercio electrónico, una vez se haya celebrado el correspondiente contrato electrónico, le corresponde a las agencias de viajes o empresas turísticas que ofrezcan esta posibilidad a través de su página web cumplir con la obligación de confirmar la recepción de la aceptación de la oferta (Guisado Moreno, p. 2004) sea enviando acuse de recibo por correo electrónico o cualquier otro medio de comunicación electrónica en las veinticuatro horas siguientes a la recepción de la aceptación; o mediante el empleo de otro medio equivalente al utilizado en el procedimiento de contratación, de forma inmediata, siempre que dicha confirmación pueda ser archivada por su destinatario.

La obligación de confirmar la recepción de la aceptación se contempla como una medida de garantía e incluso como medio de prueba de la existencia del negocio jurídico. La recepción de la aceptación se vincula con el momento en el que ha de entenderse perfeccionado el contrato por medios electrónicos. En este sentido, la disposición adicional cuarta de la Ley 34/2002 de servicios de la Sociedad de la Información y del Comercio 
electrónico establece que la conclusión del contrato celebrado a distancia tendrá lugar cuando el oferente conozca la aceptación o desde que habiéndola remitido el aceptante no puede ignorarla sin faltar a la buena fe (Arts. 1262 CC. y 54 Ccom).

Por otro lado, la contratación de servicios turísticos por medios electrónicos incorpora condiciones generales que se incorporan al contrato por imposición de organizadores o detallistas limitando la voluntad de los contratantes. En este sentido, la Ley 34/2002 de Servicios de la Sociedad de la Información y del Comercio electrónico establece la exigencia de que el prestador de servicios ponga a disposición de los destinatarios, con carácter previo al inicio del procedimiento de contratación, las condiciones generales a las que se va a sujetar el contrato electrónico, de manera que éstas puedan ser almacenadas y reproducidas. Además será necesario que el adherente manifieste su conocimiento por medio de la aceptación de las mismas, pulsando el icono destinado al efecto.

Como resultado de la actuación de la agencia de viajes, el proveedor del servicio deberá respetar frente al turista las condiciones generales que se incluyeran en la página web de la agencia de viajes, y a la inversa, el turista quedará obligado frente al proveedor de servicios turísticos como si hubieran sido estipuladas por él mismo, siendo en esta esfera jurídica donde surtirán efectos los derechos y las obligaciones que se originen con el contrato de servicios perfeccionado entre el turista y el proveedor del servicio turístico. En este sentido, ante un incumplimiento total o parcial del servicio turístico, el turista, deberá dirigirse frente al proveedor del servicio como contraparte en el contrato, y no frente a la agencia de viajes que solo ha prestado un servicio de intermediación en los contratos celebrado a distancia y por vía electrónica consistente en realizar la contratación del servicio turístico en nombre y por cuenta del turista.

A su vez, ante un incumplimiento del turista de las obligaciones derivadas del contrato de servicios perfeccionado, el proveedor del servicio turístico no podrá dirigirse frente a la agencia de viajes exigiéndole la responsabilidad, sino que dicha responsabilidad únicamente podrá exigírsela al turista. En este sentido, la agencia de viajes no responderá frente al proveedor de los incumplimientos contractuales del turista, salvo que se hubiera obligado expresamente a ello. No obstante, el turista no quedará obligado frente al proveedor del servicio en todo aquello en lo que la agencia de viajes se haya excedido en la gestión del encargo, salvo que ratifique expresamente aquello en lo que se hubiera excedido (art. 1272.2 CC).

\section{CONTRATACION ELECTRÓNICA DE PAQUETES DINÁMICOS DE TURISMO}

El desarrollo de Internet y la aparición de las compañías de bajo coste ha hecho que cada vez más los consumidores se abstengan de adquirir paquetes establecidos por un tour operador, y en cambio, confeccionen ellos mismos sus vacaciones valiéndose de herramientas electrónicas que les permiten encontrar la oferta más favorable, conocidas como metabuscadores o comparadores de precios o reservando los servicios turísticos directamente con su proveedor o a través de una agencia de viajes on line. A esta nueva forma de contratación de servicios turísticos se la conoce como paquetes dinámicos de turismo o dynamic packages.

Los paquetes dinámicos no se ajustan exactamente al concepto tradicional de viaje combinado recogido en anterior normativa ya que, a diferencia de los paquetes tradicionales 
y preorganizados por las agencias de viajes, éstas nuevas combinaciones implican un mayor nivel de libertad por parte del consumidor quien combina los servicios según sus preferencias y los adquiere a diferentes operadores, o incluso, permiten que dicha combinación de servicios se produzca a partir de un proceso automático de ofertas que se conjugan directamente en Internet.

Teniendo en cuenta que tradicionalmente la labor organizativa de las agencias se ha contemplado como requisito configurador del viaje combinado, esta nuevas combinaciones han dificultado la calificación jurídica de estos nuevos servicios y han provocado una gran confusión entre los operadores turísticos y los consumidores que contrataban con una agencia de viajes on line un paquete dinámico de turismo El problema se generaba, principalmente, porque la empresa consideraba que dicha combinación no podía obtener la calificación de viaje combinado protegido debiendo reclamar el consumidor directamente ante el prestador de servicios concreto o directamente quedar desprotegido ante la inclusión de condiciones generales cláusulas de exclusión de responsabilidad en caso de no prestarse o de prestarse defectuosamente alguno de los servicios contratados, convirtiendo su intervención como de simple intermediación.

Hasta el año 2015 no se disponía de una regulación actualizada de los paquetes dinámicos de turismo ya que ni la Directiva 90/314/CEE, de 13 de junio, relativa a los viajes combinados, relativa a los viajes combinados, las vacaciones combinadas y los circuitos combinados ni el Título IV del Real Decreto Legislativo 1/2007, de 16 de noviembre, por el que se aprueba el texto refundido de la Ley General para la Defensa de los Consumidores y Usuarios y otras leyes complementarias, tras su modificación por la Ley $3 / 2014$, de 27 de marzo (artículos 150 a 165 TRLCU) la contemplan. Esta indeterminación generaba gran confusión entre los operadores turísticos y los consumidores que contrataban con una agencia de viajes on line un paquete dinámico de turismo ante la inclusión de condiciones generales cláusulas de exclusión de responsabilidad en caso de no prestarse o de prestarse defectuosamente alguno de los servicios contratados, convirtiendo su intervención como de simple intermediación.

Ante la clara evidencia de contar con un régimen insuficiente de protección de los viajeros en el ámbito de los paquetes dinámicos turísticos, el legislador comunitario ha aprobado la reciente Directiva 2015/2302, de 25 de noviembre, relativa a los viajes combinados y a los servicios de viaje vinculados, por la que se modifican el Reglamento (CE) no 2006/2004 y la Directiva 2011/83/UE del Parlamento Europeo y del Consejo y por la que se deroga la Directiva 90/314/CEE del Consejo. No obstante, esta regulación no será de aplicación hasta el 1 de julio de 2018, ya que se ha concedido a los Estados miembros un plazo, hasta el 1 de enero de 2018, para que lleven a cabo la transposición de dicha normativa adoptando las disposiciones legales, reglamentarias y administrativas necesarias para dar cumplimiento a lo establecido en la presente Directiva (Berenguer Albadalejo, 2016, p. 33).

La presente Directiva tiene, por tanto, por objeto adaptar el alcance de la protección para tener en cuenta esta evolución, aumentar la transparencia y la seguridad jurídica de los viajeros y empresarios. Por medio de esta nueva normativa se pretende acabar con esta incertidumbre que afecta tanto a consumidores como a usuarios ya que las consecuencias de la calificación de una combinación de servicios de viajes pueden ser distintas sobre todo 
desde el punto de vista de las obligaciones a asumir por el empresario desde el punto de vista de la protección del consumidor.

Se pretende por este medio contribuir al buen funcionamiento del mercado interior y a la consecución de un nivel de protección de los consumidores elevado y lo más uniforme posible mediante la aproximación de determinados aspectos de las disposiciones legales, reglamentarias y administrativas de los Estados miembros en relación con los contratos entre viajeros y empresarios relativos a viajes combinados y a servicios de viaje vinculados, dotando de una mayor cobertura jurídica a los consumidores que contratan paquetes dinámicos de turismo. No obstante, este objetivo dependerá, en parte, de cómo se lleve a cabo la transposición de dicha normativa por las disposiciones de los Estados correspondientes.

La modernización de la Directiva se ha llevado a cabo para dar cobertura tanto a los viajes combinados comercializados por un único operador como por los comercializados por varios operadores bajo una misma calificación de viajes combinados preestablecidos. Asimismo, bajo la protección de esta nueva normativa también se han incluido una serie de combinaciones que reciben la denominación de "servicios de viaje vinculados" que se caracterizan porque son prestados por varios operadores. A pesar de que éstos últimos no se someten al mismo régimen de protección que el viaje combinado, gozan en la nueva normativa de un régimen de protección que determina la responsabilidad de cada prestador de servicios respecto de la prestación del servicio correspondiente.

\subsection{Calificación jurídica del paquete dinámico de turismo}

El término "paquete" alude a la confección de dos o más servicios turísticos cuya adquisición se hace en una misma operación, sin importar que por éstos, el viajero pague un precio global. Por su parte, el término "dinámico" alude al amplio grado de decisión que tiene el viajero para elegir los servicios que van a componer su paquete. En consecuencia, por "paquete dinámico de turismo" se entiende aquel producto compuesto por dos o más servicios turísticos que son confeccionados y adquiridos por un consumidor a través de uno o varios portales web, siempre que exista un vínculo comercial entre ellos (Camargo Gómez, 2014, p. 99).

Desde este punto de vista, la contratación electrónica de un paquete dinámico de turismo se puede hacer bien dentro del portal web de una agencia de viajes on line, en donde el viajero confecciona y adquiere los servicios sin ser adquirido a ningún otro portal, o bien en los sitios web de cada proveedor del servicio siempre que entre éstos exista un acuerdo comercial, ya que existe un paquete cuando los servicios se adquieren en un único punto de venta en el mismo proceso de reserva a raíz de una oferta o pago global. En caso de inexistencia de acuerdo entre los proveedores de los servicios turísticos, la contratación constituye una forma de adquisición de servicios turísticos sueltos, con lo que resultará de aplicación a cada contrato el régimen general.

Teniendo en cuenta esta definición anterior, el paquete dinámico de turismo se caracteriza, en primer lugar, porque está compuesto por una pluralidad de servicios turísticos. El objeto del paquete puede consistir en un servicio de transporte, un servicio de alojamiento o el alquiler de turismos o cualquier otro servicio no accesorio de éstos que constituyan una parte significativa del paquete. Puesto que las normas no determinan 
cuando un servicio es accesorio o principal, para establecer la calificación del servicio de viaje deberá tenerse en cuenta su factor funcional, es decir, si el servicio resulta autónomo o independiente con respecto al transporte, el alojamiento o el alquiler de coche. Sin embargo, lo más relevante es que por medio del paquete dinámico el turista adquiera dos o más servicios turísticos.

En segundo lugar, el paquete dinámico de turismo se caracteriza porque la combinación de los servicios turísticos sueltos es efectuada por el turista en el mismo proceso de contratación. A diferencia de lo que ocurre con el tradicional paquete preestablecido, el paquete dinámico no requiere la intervención de un organizador ni la celebración de subcontratos con los correspondientes proveedores de servicios para poder ser adquirido. Por último, los servicios turísticos que componen el paquete dinámico de turismo deben ser adquiridos a un mismo operador y, en el caso de que sean más de uno, debe existir un vínculo comercial entre ellos. Debido a que el viajero puede adquirir el paquete de servicios dentro de la misma página web donde ha combinado la pluralidad de los servicios turísticos que componen el paquete o en la página web de cada proveedor de servicios, en este segundo caso, el único requisito es que debe existir entre los proveedores algún tipo de relación comercial que permita observar la presencia de un "conjunto unitario" de servicios de viaje (Camargo Gómez, 2014, p.101).

El paquete dinámico de turismo comparte con la contratación de servicios turísticos sueltos la amplia capacidad de elección que goza el viajero para configurar la prestación de un servicio de viaje, pero se diferencia, en cambio en que en el paquete dinámico se contratan varios servicios turísticos en el mismo proceso de contratación cuando exista una asociación entre ellos. Por otra parte, el paquete dinámico se asemeja al viaje combinado en que se adquiere una pluralidad de servicios turísticos en una misma operación y el viaje sobrepasa las 24 horas de duración, pero se diferencia, sin embargo, en que no es obligatorio que una de las partes contratantes sea necesariamente una agencia de viajes, ya que el viajero puede adquirir de los proveedores directos del servicio un paquete dinámico siempre que exista (Berenguer Albadalejo, 2016, p. 36).

El mercado descrito ha evolucionado considerablemente desde la adopción de la Directiva 90/314/CEE. Además de las cadenas de distribución tradicionales, internet se ha convertido en un medio cada vez más importante a través del que se ofrecen o venden servicios de viaje. Los servicios de viaje no solo se combinan en forma de viajes combinados preestablecidos tradicionales, sino que con frecuencia se combinan a medida. Muchas de esas combinaciones de servicios de viaje se encuentran en una situación de indefinición jurídica o no están claramente incluidos dentro del ámbito de aplicación de la Directiva 90/314/CEE. Por esa razón era menester adaptar el marco legislativo a la evolución del mercado para adecuarlo mejor al mercado interior, eliminar ambigüedades y colmar las lagunas legislativas.

A ello responde la presente Directiva 2015/2302, de 25 de noviembre, relativa a los viajes combinados y a los servicios de viaje vinculados, que tiene por objeto adaptar el alcance de la protección para tener en cuenta esta evolución, aumentar la transparencia y la seguridad jurídica de los viajeros y empresarios. Esta norma incorpora nuevos elementos en el concepto de viaje combinado que no estaban comprendidos ni en la normativa comunitaria anterior sobre viaje combinado. No se aplica, sin embargo, a los viajes combinados y los servicios de viajes combinados de duración inferior a 24 horas, a menos 
que se incluya la pernoctación, a los viajes combinados que se ofrezcan, y los servicios de viaje vinculados que se faciliten, de manera ocasional y sin ánimo de lucro únicamente a un grupo limitado de viajeros.

El artículo 2 de la Directiva 2015/2302 dispone que la misma se aplica a dos tipos de combinaciones: a los "viajes combinados" ofrecidos para la venta o vendidos por empresarios a viajeros y a los denominados "servicios de viajes vinculados" que son facilitados por varios empresarios a los viajeros, y ello con independencia de la contratación se lleve a cabo de manera presencial o en línea. La norma se encarga de definir ambos tipos de servicios de una forma diferente a como estaba hasta la fecha configurado el tradicional concepto de viaje combinado.

En el ámbito de las definiciones, la Directiva 2015/2302 señala que se entenderá por "servicio de viaje": "a) el transporte de pasajeros; b) el alojamiento cuando no sea parte intrínseca del transporte de pasajeros y no tenga fines residenciales; c) el alquiler de turismos, otros vehículos de motor en el sentido del artículo 3, punto 11, de la Directiva 2007/46/CE del Parlamento Europeo y del Consejo, o motocicletas que requieran un permiso de conducción de categoría A con arreglo a lo dispuesto en el artículo 4, apartado 3, letra c) de la Directiva 2006/126/CE del Parlamento Europeo y del Consejo; d) cualquier otro servicio turístico que no forme parte intrínseca de un servicio de viaje de entre los definidos en las letras a), b) o c)" (art. 3.1 Directiva 2015/2302).

En cuanto al "viaje combinado" la Directiva 2015/2302 señala que se entiende por tal "la combinación de al menos dos tipos de servicios de viaje a efectos del mismo viaje o vacación, si esos servicios: a) son combinados por un solo empresario, incluso a petición o según la selección del viajero, antes de que se celebre un contrato único por la totalidad de los servicios, o b) con independencia de la celebración de contratos distintos con diferentes prestadores de servicios de viaje, esos servicios i) son contratados en un único punto de venta y han sido seleccionados antes de que el viajero acepte pagar, ii) son ofrecidos o facturados a un precio a tanto alzado o global, iii) son anunciados o vendidos como "viaje combinado" o bajo una denominación similar, iv) son combinados después de la celebración de un contrato en virtud del cual el empresario permite al viajero elegir entre una selección de distintos tipos de servicios de viaje, o v) son contratados con distintos empresarios a través de procesos de reserva en línea conectados en los que el nombre del viajero, sus datos de pago y su dirección de correo electrónico son transmitidos por el empresario con el que se celebra el primer contrato a otro u otros empresarios, con el o los que se celebra un contrato a más tardar 24 horas después de la confirmación de la reserva del primer servicio de viaje" (art. 3.1 Directiva 2015/2302).

Además, a diferencia de la reglamentación anterior, la reciente Directiva 2015/2302, de 25 de noviembre, relativa a los viajes combinados y a los servicios de viaje vinculados define los "servicios de viajes vinculados" como aquellos en los que "al menos dos tipos diferentes de servicios de viaje contratados para el mismo viaje o vacación, para los que se celebren contratos distintos con cada uno de los prestadores de servicios de viaje, si un empresario facilita: a) con ocasión de una única visita o contrato con su punto de venta la selección y pago por separado de cada servicio de viaje por parte de los viajeros o b) de manera específica, la contratación con otro empresario de como mínimo un servicio de viaje adicional siempre que se celebre un contrato con este otro empresario a más tardar 24 
horas después de la confirmación de la reserva del primer servicio de viaje. Cuando se adquiera no más de un tipo de servicio de viaje a que se refiere el punto 1 , letras a), b) o c), y uno o varios de los servicios turísticos de viaje a que se refiere el punto 1 , letra d), no constituirán servicios de viaje vinculados si los segundos no representan una proporción significativa del valor combinado de los servicios y no se anuncian como una característica esencial de la combinación o no constituyen por alguna otra razón una característica esencial del viaje o vacación" (art. 3.5 Directiva 2015/2302).

En este sentido, los viajes combinados deben distinguirse de los servicios de viaje vinculados, en los que los empresarios facilitan de manera presencial o en línea a los viajeros la contratación de servicios de viaje, llevándoles a celebrar contratos con distintos prestadores de servicios de viaje, inclusive mediante procesos de reserva conectados, que no presentan las características de los viajes combinados y a los que no sería apropiado imponer todas las obligaciones exigibles a estos últimos.

Procede aclarar que constituyen viajes combinados los servicios de viaje que se combinan después de la celebración de un contrato en virtud del cual un empresario permite a un viajero elegir entre una selección de diferentes tipos de servicios de viaje, como es el caso de una "caja regalo" para un viaje combinado. Además, una combinación de servicios de viaje debe considerarse un viaje combinado cuando el nombre, los datos de pago y la dirección de correo electrónico del viajero se transfieran entre los empresarios y cuando se celebre otro contrato a más tardar transcurridas 24 horas desde que se confirmó la reserva del primer servicio de viaje.

Conforme a las definiciones de viaje combinado y de servicio de viaje combinadas incorporadas por la Directiva 2015/2302, de 25 de noviembre, relativa a los viajes combinados y a los servicios de viaje vinculados, el paquete dinámico de turismo cumple las características señaladas en el concepto de servicios de viajes combinados en el caso de que el paquete lo contrate el turista en los sitios web de cada proveedor del servicio siempre que entre éstos exista un acuerdo comercial, ya que existe un paquete cuando los servicios se adquieren en un único punto de venta en el mismo proceso de reserva a raíz de una oferta o pago global. (Camargo Gómez, 2014, p. 107.).

\subsection{Partes intervinientes en el contrato de paquetes dinámicos}

A quien vende un paquete dinámico se le denomina operador turístico, esto, es cualquier persona que actúe con fines relacionados con su actividad comercial, negocio, oficio o profesión. Este puede ser una agencia de viajes on line o también un proveedor de servicios turísticos (aerolíneas, hoteles, arrendadores de coches, etc.). No obstante, uno y otro caso tendrán la condición de parte contractual dependiendo de la forma como el turista contrate el paquete dinámico.

Cuando el turista adquiere un paquete directamente con una agencia de viajes por Internet, ésta será parte contractual del paquete dinámico de turismo, con independencia de si se vale de medios propios o cuenta con la intervención de un proveedor de servicios para ejecutar el contrato. Si bien el proveedor directo de los servicios se involucra en el cumplimiento principal que dimana del contrato, lo hace de forma indirecta debido a que entre este y el consumidor no existe relación jurídica alguna. Por lo tanto, en este caso, la agencia de viajes on line será quien deba de responder por la no prestación o la prestación defectuosa de alguno de los servicios contratados (Camargo Gómez, 2014, p. 110). 
En cambio, en el caso de que el viajero adquiera una pluralidad de servicios celebrando contratos separados en el portal web de cada uno de los prestadores del servicio, pero utilice un único punto de venta en el mismo proceso de reserva y los servicios sean facturados a un precio global, o pago por separado pero el empresario facilite el único punto de venta, y sean anunciados y/o vendidos como paquete dinámico o adquiridos a través de procesos en línea conexos, será considerada parte contractual cada proveedor del servicio, aunque habrá de tener en cuenta si en dicha contratación interviene el organizador de los diferentes servicios turísticos que compone el paquete, si entre los prestadores de los servicios existe un vínculo comercial entre ellos y se ha efectuado en el mismo proceso de contratación.

De conformidad con lo establecido en la reciente Directiva 2015/2302, de 25 de noviembre, relativa a los viajes combinados y a los servicios de viaje vinculados se entiende por "empresario" toda persona física o toda persona jurídica, ya sea de titularidad privada o pública, que actúe, incluso a través de otra persona que obre en su nombre o siguiendo sus instrucciones, con un propósito relacionado con su actividad comercial, empresa, oficio o profesión en relación con contratos regulados por la presente Directiva, tanto si actúa como organizador, minorista, empresario que facilita servicios de viaje vinculados o como prestador de servicios de viaje (art. 3.7 Directiva 2015/2302)

Asimismo, la Directiva señala que se entiende por "organizador": un empresario que combina y vende $u$ ofrece viajes combinados, directamente o a través de otro empresario $o$ junto con él, o el empresario que transmite los datos del viajero a otro empresario cuando se cumplan los requisitos establecidos para el caso de que se contrate con diferentes prestadores de servicios de viaje. Y se entenderá por "minorista": empresario distinto del organizador que vende $u$ ofrece viajes combinados compuestos por un organizador (art. 3.8 y 9 Directiva 2015/2302).

Por otra parte, el viajero es toda persona que tiene la intención de celebrar un contrato o tiene derecho a viajar con arreglo a un contrato celebrado bajo la forma de paquete dinámico, incluidos los viajeros de negocios en la medida en que no viajen sobre la base de un contrato marco con un operador especializado en la organización de viajes de negocios. En este sentido, la Directiva 2015/2302, de 25 de noviembre, relativa a los viajes combinados señala que "viajero" es toda persona que tiene la intención de celebrar un contrato o tiene derecho a viajar con arreglo a un contrato de viaje combinado o de viaje vinculado, de conformidad con lo establecido en dicha norma comunitaria (art. 3.6 Directiva 2015/2302).

\subsection{Régimen de responsabilidad en el paquete dinámico de turismo}

Al abordar la cuestión de la calificación jurídica del paquete dinámico de turismo ya se aludió a la confusión que se genera respecto de esta modalidad de prestación de servicios turísticos a la hora de determinar la responsabilidad de los operadores de servicios turísticos que intervienen en la ejecución del contrato. Esta indeterminación jurídica ha sido en parte resuelta por la Directiva 2015/2302, de 25 de noviembre, relativa a los viajes combinados y a los servicios de viaje vinculados (arts. 13 a 16 Directiva 2015/2302) que señala las directrices que deben de tener en cuenta los Estados miembros a la hora de implementar dicha normativa a través de sus legislaciones nacionales. 
En relación con el régimen de responsabilidad, la Directiva 2015/2302, de 25 de noviembre, señala respecto de la ejecución de los viajes combinados que los Estados miembros que implementen dicha normativa "garantizarán que el responsable de la ejecución de los servicios de viaje incluidos en el contrato de viaje combinado sea el organizador, con independencia de que estos servicios vayan a ser ejecutados por el organizador o por otros prestadores de servicios de viaje. Los Estados miembros podrán mantener o establecer en su Derecho nacional disposiciones que estipulen que también el minorista es responsable de la ejecución del viaje combinado" (art. 13 Directiva 2015/2302).

En este sentido, se establece que el viajero informará al organizador sin demora indebida, teniendo en cuenta las circunstancias de cada caso, de cualquier falta de conformidad que observe durante la ejecución de un servicio de viaje incluido en el contrato de viaje combinado. Si cualquiera de los servicios del viaje no se ejecuta de conformidad con el contrato de viaje combinado, el organizador deberá subsanar la falta de conformidad, salvo: a) si resulta imposible, o b) si ello entraña un coste desproporcionado, teniendo en cuenta la gravedad de la falta de conformidad y el valor de los servicios del viaje afectados. Además, sin perjuicio de las excepciones previstas en el apartado 3, si el organizador no subsana la falta de conformidad en un plazo razonable establecido por el viajero, el propio viajero podrá hacerlo y solicitar el reembolso de los gastos necesarios. No será necesario que el viajero especifique un plazo si el organizador se niega a subsanar la falta de conformidad o si se precisa una solución inmediata (Berenguer Albadalejo, 2016, p. 46).

La Directiva establece normas específicas en materia de vías de recurso en lo que respecta a la falta de conformidad en la ejecución del contrato de viaje combinado. El viajero debe tener derecho a que se resuelvan sus problemas y, cuando una proporción significativa de los servicios de viaje incluidos en el contrato de viaje combinado no pueda prestarse, el organizador ofrecerá, sin coste adicional alguno para el viajero, fórmulas alternativas adecuadas, de ser posible de calidad equivalente o superior a las especificadas en el contrato, para la continuación del viaje combinado, también cuando el regreso del viajero al lugar de salida no se efectúe según lo acordado. Cuando las fórmulas alternativas propuestas representen un viaje combinado de menor calidad que la especificada en el contrato de viaje combinado, el organizador aplicará al viajero una reducción adecuada del precio. Por su parte, el viajero solo podrá rechazar las fórmulas alternativas propuestas si no son comparables a lo acordado en el contrato de viaje combinado o si la reducción de precio concedida es inadecuada (Camargo Gómez, 2014, p. 110).

Cuando una falta de conformidad afecte sustancialmente a la ejecución del viaje combinado y el organizador no la haya subsanado en un plazo razonable establecido por el viajero, este podrá poner fin al contrato de viaje combinado sin pagar ninguna penalización por terminación y solicitar, en su caso, una reducción del precio y/o una indemnización por daños y perjuicios. Si no es posible encontrar fórmulas alternativas o el viajero rechaza las fórmulas alternativas propuestas, este tendrá derecho a una reducción del precio y/o a una indemnización por daños y perjuicios sin poner fin al contrato de viaje combinado. Si el viaje combinado incluye el transporte de pasajeros, el organizador, en los casos indicados en los párrafos primero y segundo, repatriará además al viajero en un transporte equivalente sin dilaciones indebidas y sin coste adicional para este.

Por otra parte, si es imposible garantizar el retorno del viajero según lo convenido en el contrato de viaje combinado debido a circunstancias inevitables y extraordinarias, el 
organizador asumirá el coste del alojamiento que sea necesario, de ser posible de categoría equivalente, por un período no superior a tres noches por viajero. Cuando la legislación de la Unión sobre derechos de los pasajeros aplicables a los correspondientes medios de transporte para el regreso del viajero establezca períodos más largos, se aplicarán dichos períodos. La limitación de los costes a que se refiere el apartado 7 del presente artículo no se aplicará a las personas con movilidad reducida, tal como se definen en el artículo 2, letra a), del Reglamento (CE) no 1107/2006, y a sus acompañantes, las mujeres embarazadas y los menores no acompañados, así como a las personas con necesidad de asistencia médica específica, si sus necesidades particulares han sido notificadas al organizador, al menos 48 horas antes del inicio del viaje combinado.

Además la Directiva 2015/2302, de 25 de noviembre, señala la posibilidad de ponerse en contacto con el organizador a través del minorista cuando establece que "los Estados miembros garantizarán que el viajero pueda enviar directamente al minorista a través del cual fue contratado el viaje combinado mensajes, peticiones o quejas en relación con la ejecución del viaje combinado. El minorista transmitirá sin demora indebida dichos mensajes, peticiones o quejas al organizador. A efectos del cumplimiento de términos o de plazos de prescripción, el acuse de recibo por el minorista de los mensajes, peticiones o quejas a que se refiere el párrafo primero se considerará acuse de recibo por el organizador" (art. 15 Directiva 2015/2302).

Por último, la Directiva 2015/2302, de 25 de noviembre, incluye la obligación de prestar asistencia a los viajeros cuando señala que "Los Estados miembros garantizarán que el organizador proporcione asistencia adecuada y sin demora indebida al viajero en dificultades, en particular mediante: a) el suministro de información adecuada sobre los servicios sanitarios, las autoridades locales y la asistencia consular, y b) la asistencia al viajero para establecer comunicaciones a distancia y la ayuda para encontrar fórmulas de viaje alternativas. El organizador podrá facturar un recargo razonable por dicha asistencia si la dificultad se ha originado intencionadamente o por negligencia del viajero. Dicho recargo no superará en ningún caso los costes reales en los que haya incurrido el organizador (art. 16 Directiva 2015/2302).

Dadas las diferencias entre los Derechos nacionales y entre las prácticas en cuanto a las partes en los contratos de viaje combinado y a la recepción de los pagos realizados por los viajeros o en su nombre, los Estados miembros deben poder exigir a los minoristas que también estén protegidos frente a la insolvencia. Con esta finalidad, la nueva Directiva establece normas a fin de evitar que las obligaciones de protección frente a la insolvencia supongan un obstáculo a la libre circulación de servicios y a la libertad de establecimiento. Por lo tanto, los Estados miembros quedan obligados a reconocer la protección frente a la insolvencia con arreglo a la legislación del Estado miembro de establecimiento. Para facilitar la cooperación administrativa y el control de los organizadores y, si ha lugar, de los minoristas que operan en distintos Estados miembros en relación con la protección frente a la insolvencia, los Estados miembros están asimismo obligados a designar puntos de contacto centrales.

En relación con los servicios de viajes vinculados, la Directiva 2015/2302, de 25 de noviembre, establece, por un lado, que "Los Estados miembros velarán por que los empresarios que faciliten servicios de viajes vinculados constituyan una garantía para el 
reembolso de todos los pagos que reciban de los viajeros, en la medida en que un servicio de viaje que forme parte de unos servicios de viaje vinculados no se ejecute como consecuencia de la insolvencia del empresario. Si dichos empresarios son la parte responsable del transporte de pasajeros, la garantía cubrirá también la repatriación de los viajeros. Se aplicarán, mutatis mutandis, el artículo 17, apartado 1, el artículo 17, apartados 2 a 5, y el artículo 18" (art. 19.1 Directiva 2015/2302).

Por otra parte, respecto de la información que se debe suministrar en la prestación de servicios vinculados, la Directiva 2015/2302, de 25 de noviembre, establece que "antes de que el viajero quede obligado por cualquier contrato que dé lugar a la elaboración de unos servicios de viaje vinculados, o por cualquier oferta correspondiente, el empresario que facilita servicios de viaje vinculados, incluso si no está establecido en un Estado miembro pero destina, por cualquier medio, tales actividades a un Estado miembro, indicará de forma clara, comprensible y destacada: a) que el viajero no podrá acogerse a ninguno de los derechos que se aplican exclusivamente a los viajes combinados en virtud de la presente Directiva y que cada prestador de servicios será el único responsable de la correcta prestación contractual de su servicio, y b) que el viajero gozará de la protección frente a la insolvencia contemplada en el apartado 1" (art. 19.2 Directiva 2015/2302).

De esta forma se aclara, en parte, el régimen de responsabilidad aplicable que va asumir la agencia de viajes on line cuando vende un viaje combinado a través de su página web que vincula los diferentes prestadores del servicio, ya que ésta asumirá las responsabilidades que se deriven de la ejecución de los servicios de viaje incluidos en el contrato de viaje combinado cuando asuma la condición de organizador, con independencia de que estos servicios vayan a ser ejecutados por el organizador o por otros prestadores de servicios de viaje. Por otra parte, en el caso de la prestación de servicios de viaje vinculados, cada prestador de servicios será el único responsable de la correcta prestación contractual de su servicio.

\section{CONCLUSIONES}

Todo oferente o intermediario en la comercialización de servicios turísticos on line realizan una actividad de prestación de servicios de la sociedad de la información y por tanto deberá de prestar la información requerida para llevar a cabo su correcta identificación frente al usuario de dicho servicio. En este sentido, las disposiciones sobre comercio electrónico imponen a los prestadores de servicios de la sociedad de la información que utilicen Internet como cauce publicitario para ofertar sus productos o servicios, el deber de suministrar a los destinatarios una serie de deberes de información con la finalidad de que éstos tengan la suficiente información sobre la agencia y sobre las características del producto o servicio que se pretende adquirir que si contratasen con una agencia de viajes que operase por medios tradicionales.

En la práctica, cada vez es más frecuente la contratación de un servicio turístico suelto consistente en la compra de un billete de avión o tren o en la reserva de una plaza de alojamiento por separado a través de las páginas web de las agencias de viajes que operan en Internet. Cuando la contratación electrónica del servicio turístico se efectúe entre el turista y el prestador del servicio a través de la página web de una agencia de viajes on line, la agencia de viajes realiza labores de intermediación entre el turista y el proveedor final del servicio turístico, celebrándose en este caso dos relaciones jurídicas: la que se celebra entre 
el turista con la agencia de viajes on line y la que se celebra entre el turista con el proveedor del servicio.

Como novedad, se encuentra en la reciente regulación sobre viajes combinados establecida por la reciente Directiva 2015/2302, de 25 de noviembre, relativa a los viajes combinados y a los servicios de viaje vinculados, por la que se modifican el Reglamento (CE) no 2006/2004 y la Directiva 2011/83/UE del Parlamento Europeo y del Consejo y por la que se deroga la Directiva 90/314/CEE del Consejo. Entre los elementos incorporados en la nueva regulación destacan la responsabilidad frente al consumidor de cuantas empresas concurran conjuntamente en el contrato de viajes combinados lo que implica que tanto la agencia mayorista como la agencia minorista responderán de manera solidaria ante el cliente. En este sentido, y a partir de la promulgación de esta normativa, la compra de paquetes turísticos por Internet gozará de las mismas garantías que los que puedan adquirirse en una agencia física.

Complementariamente se obliga a los empresarios que faciliten servicios de viaje vinculados a informar a los viajeros de que no están contratando un viaje combinado y de que los prestadores de servicios únicamente son responsables de la correcta ejecución de sus contratos. Los empresarios que faciliten servicios de viaje vinculados deben, además, prestar protección frente a la insolvencia para la devolución de los pagos que perciban y, en la medida en que sean responsables del transporte de pasajeros, para la repatriación de estos, y deben informar a los viajeros en consecuencia.

\section{REFERENCIAS BIBLIOGRÁFICAS}

Aurioles Martín, A. (2006). Introducción al derecho turístico, Madrid: Tecnos.

Berenguer Albadalejo, C. (2016). "Luces y sombras de la nueva Directiva (UE) 2015/2302 del Parlamento Europeo y del Consejo, de 25 de noviembre de 2015, relativa a los viajes combinados y a los servicios de viaje vinculados" International Journal of Scientific Management and Tourism, April 2016, 33-50.

Batuecas Caletrío, A., Aparicio Vaquero, J. P.; (2013). "La contratación on line de servicios turísticos" en Paniza Fullana, A., Nuevas formas de contratación on line de servicios turísticos: subsunción en los tipos leales y distribución de responsabilidad, Comares, Granada, 49-78.

Carmago Gómez, J. D (2014). “Contratación electrónica de paquetes dinámicos de turismo en ordenamiento jurídico español", Ars luris Salmanticensis, Vol 2, Diciembre 2014, 95125.

Cavanillas Mugica, S. (2001). Turismo y Comercio electrónico. La promoción y contratación "on line" de servicios turísticos, Granada: Comares.

Cruz Rivero, D. (2009). “Contratación electrónica con consumidores”, RCE, 109, 3-42.

Flaquer Ruitort, J. (2015). "Algunos problemas jurídicos derivados de las nuevas formas de comercializacion hotelera a través de canales electrónicos", Revista Aranzadi de Derecho y Nuevas Tecnologías, num. 37, Enero-Abril, 119-133.

González de la Alaiza Cardona, J. J. (2010). Tratado de Contratos, Tirant lo Blanch, Valencia. Guisado Moreno, A. (2004). Formación y perfección del contrato en Internet, Marcial Pons, Madrid, 154-156. 
Márquez Lobillo, P. (2011). "El consumidor en la contratación electrónica de servicios turísticos", RDM, 282., octubre-diciembre, 209-242.

Melgosa Arcos, F. J. (2011). "Directiva de Servicios y Turismo: aproximación a la incidencia de la Directiva de Servicios en marco jurídico del turismo español" Noticias de la Unión Europea, 317, junio, 47-72.

Miguel Asensio, P.A. (2011). Derecho Privado de Internet, Aranzadi, Cizur Menor.

Muñoz Gil, C. (2011). “Contratación y servicios turísticos: especial estudio de la legislación valenciana sobre turismo", Diario la Ley, 7551, 1-17.

Paniza Fullana, A. (2012). "La protección del adquirente a distancia de servicios turísticos", Revista de Derecho Privado, 2, 15.

Para citar este artículo: Asensi, A. (2016). Contratación on line de servicios turísticos y paquetes dinámicos de turismo. Investigaciones Turísticas, (12), 163-182. http://dx.doi.org/10.14198/INTURI2016.12.08 